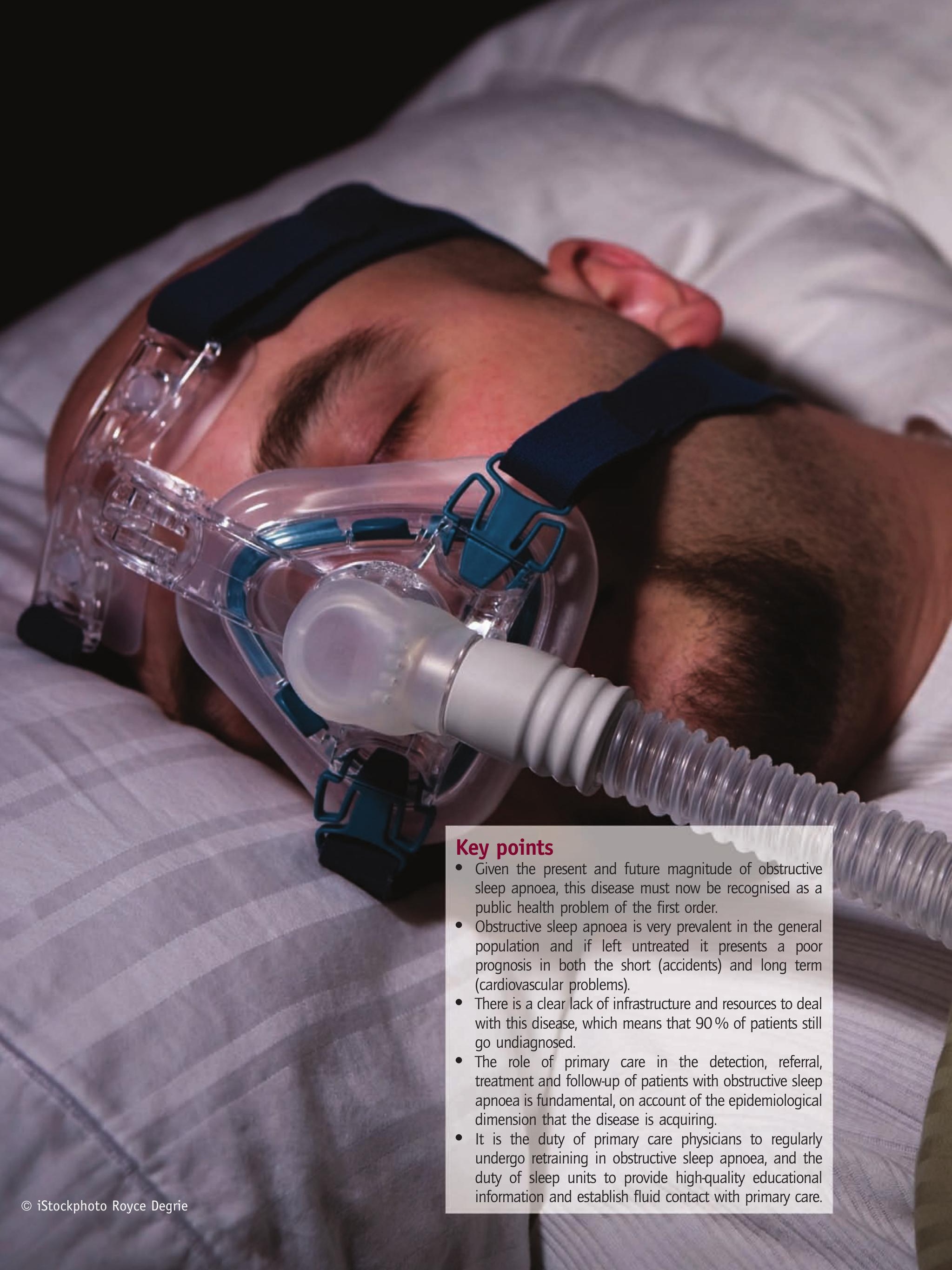




\section{Is sleep apnoea a specialist condition? The role of general practitioners}

\section{Educational aims}

1 To identify obstructive sleep apnoea (OSA) as a public health problem of the first order that requires involvement at all levels of care, from primary to specialist.

1 To pay special attention to aspects of OSA less familiar to primary care, particularly its relationship with cardiovascular diseases.

1 To analyse the expected role of primary care in the diagnosis, treatment and follow-up of patients with OSA and its risk factors.

1 To make clear that treatment with continuous positive airway pressure (CPAP) is not the exclusive domain of specialist care, as many of the problems associated with OSA can be monitored in primary care.

\section{Summary}

There is no doubt that OSA is a public health problem, on account of its great prevalence and significant negative effects on health, in both the short and long term. These effects stem from the association observed between OSA and traffic and workplace accidents, as well as its association with various cardiovascular pathologies and diseases. The growing number of patients being diagnosed and treated makes it increasingly difficult for specialists to monitor and follow them up on their own. There is therefore a growing need to involve primary care staff in the attention given to these patients. As in the case of other public health problems such as obesity and diabetes, the primary care physician must acquire sufficient knowledge and expertise both to refer a patient with suspected osa to the suitable specialist and to conduct a basic follow-up, regardless of whether the patient has been prescribed treatment with CPAP.

\section{Obstructive sleep apnoea and its consequences}

OSA is characterised by recurring episodes of limited airflow during sleep as a result of an anatomical-functional alteration of the upper airway that leads to the latter's collapse. This decreases the oxyhaemoglobin saturation and induces arousals, resulting in unrefreshing sleep, excessive daytime sleepiness and a group of metabolic, inflammatory, neuropsychiatric, respiratory and cardiovascular disorders [1]. OSA has an estimated prevalence of $2-4 \%$ in the middle-aged general population, and this figure rises with age $[2,3]$. One of the most important aspects of OSA is its capacity to have a negative impact on a patient's quality of life [4] and to increase his or her morbidity and mortality in two basic respects: the risk of accidents [5-7] and cardiovascular consequences [8] (table 1).

\section{M.A. Martínez-García ${ }^{1,2}$}

P. Catalán Serra ${ }^{1}$

${ }^{1}$ Pneumology Unit, Requena General Hospital, Valencia, Spain ${ }^{2}$ CIBER de enfermedades respiratorias (CIBERES)

\section{Correspondence} M.A. Martínez-García Pneumology Unit Requena General Hospital Paraje Casa Blanca s/n 43230 - Valencia Spain miangel@comv.es

Competing interests None declared. 


\section{Table 1. Risk of cardiovascular com- plications or accidents in patients with untreated obstructive sleep apnoea}

\begin{tabular}{lc} 
Disease & $\begin{array}{c}\text { Odds ratio } \\
\text { (95\% CI) }\end{array}$ \\
Arterial hypertension & $1.37(1.03-1.83)$ \\
Ischaemic heart failure & $1.27(0.99-1.62)$ \\
Heart failure & $2.38(1.22-4.62)$ \\
Ischaemic stroke & $1.58(1.02-2.46)$ \\
Auricular fibrillation & $4.02(1.03-15.5)$ \\
Pulmonary hypertension & $1.4(1.1-2.8)$ \\
Diabetes mellitus & $1.7(1.1-2.7)$ \\
General mortality & $6.24(2.01-19.4)$ \\
Traffic accidents & $6.3(2.4-16.2)$ \\
Traffic accident plus alcohol & $11.2(3.8-32.9)$ \\
consumption & \\
Workplace accidents & $2.2(1.3-3.8)$ \\
\hline Data are taken from [5, 8-11]. & \\
\hline
\end{tabular}

\section{OSA and the risk of accidents}

The risk of being involved in a traffic accident is increased six-fold in patients with OSA, and furthermore this possibility is doubled in combination with alcohol consumption [5]. Similarly (although there are fewer studies in this respect), there is an increased risk of accidents in the home and workplace [9]. It has been observed, however, that treatment with CPAP is capable of reversing this situation almost to a normal level [12].

\section{OSA and cardiovascular disease}

There is an ever-growing body of evidence to show that OSA can provoke certain cardiovascular diseases or worsen their prognosis. This association seems to be based largely on physiopathological factors, as patients with OSA display almost all the classic intermediate mechanisms capable of giving rise to a cardiovascular disease: arteriosclerosis, systemic inflammation, hypercoagulability, sympathetic activation and other metabolic events [8].

\section{OSA and arterial hypertension}

This is probably the association that has been found most consistently, and studied most comprehensively. Studies with a very high level of evidence confirm, in keeping with the majority of the most widely recognised international guidelines on the subject, that OSA is an independent risk factor for arterial hypertension [13]. Furthermore, several recent meta-analyses and studies verify that treatment with CPAP is capable of significantly reducing blood pressure $[14,15]$.

\section{OSA and ictus}

Patients with OSA have a two- to threetimes increased risk of suffering from an ictus [10]; this relationship also seems to be present even in the absence of arterial hypertension, probably on account of other intermediate mechanisms such as cardiac arrhythmias, hypercoagulability and arteriosclerosis. Moreover, the presence of untreated OSA in patients who have previously experienced an ictus appears to be associated with a greater risk of suffering from a further ictus [16], as well as with impaired functional recovery [17] and greater excess mortality [18]. Some observational studies have also established that treatment with CPAP is associated with a lower risk of an ictus, a new cardiovascular event or death in patients who have previously suffered a cerebrovascular event and present an excess of sleep respiratory disturbances [19].

\section{OSA and ischaemic heart disease}

Some authors have used population studies to warn that patients with OSA present a two- to threefold increase in the prevalence of ischaemic heart disease [20]. Research on the effect of treatment with CPAP on these patients is in progress.

\section{OSA and heart failure}

OSA has a two-pronged relationship with severe heart failure. On the one hand, OSA can trigger a reduction in the ejection fraction of the left ventricle and, on the other hand, the presence of a heart failure, whether systolic or diastolic, increases the probability of central respiratory events, which, in turn, intensify the disturbance in the left ventricle, thereby closing the pathogenic vicious circle [21]. Although CPAP does not appear to improve mortality in patients with heart failure and central sleep apnoea (Cheyne-Stokes breathing), it may be effective in the evolution of patients predominantly subject to obstructive events [22].

\section{OSA and mortality}

Several studies with an extensive follow-up conclude that the presence of untreated OSA produces excess mortality, both in general and, more especially, from cardiovascular causes, 
and this seems more evident in individuals aged $<50$ yrs $[11,23]$. Treatment with CPAP seems to reduce the excess mortality observed in patients with OSA, especially when it is related to the cardiovascular system [24].

\section{OSA and quality of life}

OSA - especially in its most symptomatic forms lowers quality of life in health terms in patients of all ages, although this phenomenon is more evident in middle-aged individuals with an active social and working life [4]. Treatment of these serious forms of OSA with CPAP clearly improves patients' quality of life, to the point of attaining values similar to those of individuals of the same age and sex who do not suffer from the disease [25].

\section{Other associations}

Other diseases or cardiovascular risk factors associated with OSA are cardiac arrhythmias (unsustained ventricular arrhythmias and auricular fibrillation), pulmonary hypertension, metabolic syndrome, insulin resistance and diabetes [8].

\section{OSA as an important public health problem}

Numerous epidemiological population studies concur that OSA is very prevalent. Young et al. [2], who considered OSA an excess of sleep respiratory disturbances (apnoea-hypopnoea index $(\mathrm{AHI})>10 \mathrm{~h}^{-1}$ ) plus secondary symptoms (daytime hypersomnia), observed a prevalence of OSA of $24 \%$ in females and $4-6 \%$ in males in the general middleaged population [2]. This figure can be as high as 20\% in people aged $>65$ yrs, which gives OSA a frequency similar to those observed in the general population for diabetes and asthma [3]. It has been estimated, however, that only $5-10 \%$ of serious cases of OSA are currently diagnosed, so we are very far from reaching an acceptable level of diagnosis [26]. The prevalence of sleep-related symptoms is much higher in individuals with an excess of sleep respiratory disturbances, and, therefore, there is a risk of OSA (sleep respiratory disturbances + secondary symptoms). It has been estimated that $>25 \%$ of the middle-aged population of both sexes present an $\mathrm{AHI}>5 \mathrm{~h}^{-1}$, and $15-20 \%$ an $\mathrm{AHI}>10 \mathrm{~h}^{-1}$. These figures rise to $80 \%$ and $65 \%$, respectively, in individuals aged $>65$ yrs. Furthermore, an $\mathrm{AHI}>30 \mathrm{~h}^{-1}$, i.e. the presence of severe OSA if accompanied by symptoms, is observed in $7 \%$ of men and $3 \%$ of women in middle age (figures that can rise to $20-25 \%$ in the elderly) $[27,28]$. Finally, the prevalence of more common risk factors for OSA, apart from age, must be considered, namely: chronic snoring (present in $40 \%$ of the male population and $20 \%$ of the female, and more common with age) and obesity (present in 25\% of the general population and increasing in recent years). Other triggers or coadjuvant factors for OSA, such as smoking and treatment with benzodiazepines and/or alcohol, are very prevalent in society, [28]. The end result of all this is that many millions of people all over the world present clear risk factors for suffering from OSA, or are already suffering from this disease.

A few years ago the diagnosis of OSA was nothing more than a mere medical curiosity considered devoid of any pathological implications. It has required over 20 yrs for OSA to be recognised as a health problem of the first order $[29,30]$, regarded by some as having the same significance as the problems triggered by hypertension, diabetes and smoking. OSA satisfies each and every one of the requisites for consideration as a public health problem, namely:

1. A high prevalence in the general population $[1,2,27,28]$.

2. The capacity to provoke an increase in morbidity-mortality in both the short (accidents) and long (cardiovascular) term, as well as a negative impact on the patient's quality of life [4-8].

3. The existence of diagnostic tools sufficiently validated to discern with a significant degree of reliability which patients are susceptible to treatment, on account of their worse prognosis [31].

4. The existence of a treatment, CPAP, which has proved to be very costeffective (see 'How expensive is CPAP?') in the serious forms of the disease $[32,33]$. In this respect, even though the broad cost (direct and indirect) is difficult to calculate, untreated OSA does seem to generate enormous financial expense. It has been calculated that people with severe untreated OSA consume up to three times more health resources than those treated with CPAP or those who do not suffer from the disease. In the USA, it has been calculated that the overall cost of untreated OSA would exceed that of other chronic diseases, such as asthma (\$11bn in 1994) and chronic obstructive pulmonary
Educational questions

1. With respect to the role of the primary care physician vis à vis an OSA patient treated with CPAP, indicate the correct answer:

a) Given the great complexity of treatment with CPAP, sleep apnoea patients under this treatment must always be followed up by specialists. b) Once treatment with CPAP has been instigated, there is no need to follow up the patient, as it is very unlikely that this treatment could be withdrawn in the future.

c) The mild sideeffects of CPAP can be solved in primary care, while the more serious ones require referral to a specialist. d) The reappearance of snoring and sleepiness in a patient treated with CPAP is not sufficient reason to refer him or her to a specialist.

2. Of the following cardiovascular diseases, which presents the most scientific evidence for both a relationship with OSA and the effectiveness of CPAP?

a) Stroke

b) Pulmonary hypertension

c) Systemic arterial

hypertension

d) Ischaemic heart disease

3. With respect to referral by a primary care physician of a patient with suspicion of OSA, which of the following is not true?

a) The fact that the job of a patient with a wellfounded suspicion of sleep apnoea entails risk means that the referral should be preferential.

b) Obesity, well-controlled arterial hypertension or mere snoring with no other additional characteristics 
disease (COPD) (\$23bn in 2002), and would equal the cost for diabetes (\$132bn in 2002) $[18,19]$.

5. Trend towards increased prevalence. As obesity is the most important risk factor for OSA (sex, age, obesity, family history and genetic and craneo-facial factors are others), and as it has clearly increased to epidemic proportions in recent years, the trend in the evolution of OSA is notably upward.

There is no doubt, therefore, that OSA fulfils each and every one of the principles needed for it to be considered as a public health problem, and it is thus the duty of the healthcare collective to locate, diagnose and treat the greatest number of individuals with OSA or an excess of sleep respiratory disturbances; it is also their duty to establish guidelines for prevention and for the notification of the public about this disease, which has ceased to be a medical curiosity and become one of the disorders most widely dealt with in respiratory consulting rooms all over the world in recent years. Moreover, it is the duty of the authorities, as in other public

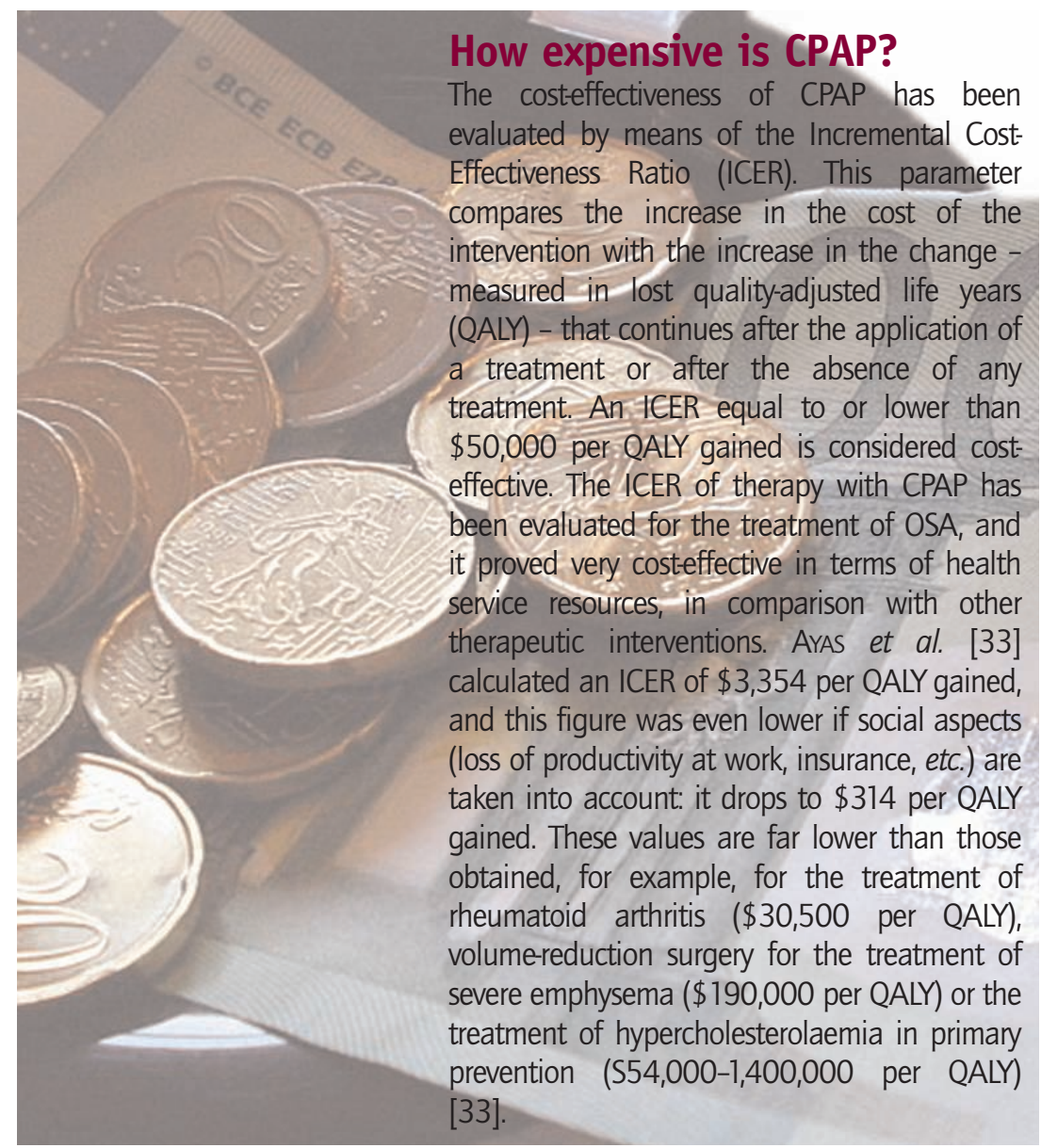

health problems, to provide accredited centres with sufficient materials and infrastructure to fulfil these functions and promote education about and prevention of this disease. Many deficiencies remain, however; these can probably be best summed up as an evident lack of resources and training of health professionals in this disease, especially in primary care.

\section{OSA in primary care: identifying the problem}

Many health systems give primary care physicians (PCPs) a preponderant role in the selection of a high percentage of the patients who go on to be diagnosed or treated in a hospital setting, as the ultimate decision to refer to a specialist rests with these doctors. It has also been calculated that up to $80 \%$ of the population is attended at some point by their PCP. This scenario therefore requires a perfect interaction between primary and specialised care, as well as the specific involvement of both in those diseases considered to be public health problems on account of their epidemiological characteristics. The functions of the PCP regarding the detection of cases and prioritisation of the referral and subsequent monitoring of patients are undoubtedly crucial to the smooth working of a health system, as has frequently been demonstrated in diseases usually considered as public health problems, such as arterial hypertension, diabetes or obesity. As a public health problem of the first order [29,30], OSA must be handled as such in the various levels of care involved, from its detection to its therapeutic monitoring. The reality falls far short of this ideal situation, however, as there is a clear lack of infrastructure, personnel and knowledge with regards to OSA in various fields, including care.

\section{Prevalence of OSA symptoms in primary care}

If, as mentioned above, OSA is a very prevalent disease, it is obvious that its risk factors, or the presence of symptoms and signs related to it, are even more widespread. The prevalence of symptoms related to OSA, and of sleep disorders in general, in patients who see their PCP for whatever reason is far higher than the prevalence of these symptoms in the overall population of the same sex and age range. This can be explained by the characteristics inherent to this sub-group 
of the population, which is generally older, more obese and subject to a greater number of comorbilities, especially of a cardiovascular and neuropsychiatric nature, all of which have been related to a greater probability of suffering from OSA. Netzer et al. [34] observed, when they analysed 744 patients attending a primary care centre, that $52.2 \%$ presented chronic snoring (in $24.6 \%$ this was extremely noisy), that $47.9 \%$ snored over three times a week and that $11.1 \%$ experienced witnessed apnoeas. The authors concluded that $37.5 \%$ of the individuals analysed presented a high clinical probability of OSA, and that $18.4 \%$ experienced sleepiness while driving and were therefore clearly candidates for referral to a sleep unit [34].

\section{Suspicion of OSA in primary care}

Despite the fact that in the past decade there has unquestionably been an overall increase in the number of diagnoses of OSA subsequently referred to sleep units [35], both from primary care and from other areas, various studies show that there is a clear failure to suspect the incidence of OSA in primary care. This is one of the reasons why $90 \%$ of patients with OSA still go undiagnosed, and why PCPs refer only a small percentage of the patients they see in their consulting room. Several determining factors seem to foment this situation. The data presented below are entirely based on research studies.

\section{Deficient compilation of a clinical sleep record}

Only $6 \%$ of the medical histories compiled in primary care make explicit reference to possible problems during sleep in a population in which, as mentioned above, $>30 \%$ of individuals present risk factors for OSA. Only 10\% of PCPs ask at least two questions related to sleep in standardised patients at a normal appointment covered by researchers [36]. NAMEN et al. [37] observed that, despite a written or verbal note from the researchers to include data concerning the quantity or quality of their patients' sleep, staff in primary care did not collect data on sleep-related diseases in $>80 \%$ of individuals, even though they did collect, spontaneously and without any need for a reminder, data on smoking and alcohol. Furthermore, even in the $15-20 \%$ of medical histories that did feature sleep-related data, only $11 \%$ of these ( $1 \%$ of the overall total) considered the data collected worthy of examination and therefore integral to a doctor's arrival at a diagnosis. Virtually none of the histories presented any written advice or treatment related to sleep in general, or sleep apnoea in particular, and once again this percentage was much lower than that observed for other public health problems such as obesity and smoking. This failure to collect data or give therapeutic advice was equally apparent with individuals with a high clinical probability of suffering from OSA. The failure to collect data was especially striking in the case of snoring and led the authors to deduce that the PCPs do not consider this phenomenon to be a potentially pathological entity [37].

\section{Referral of only the most typical forms of the disease}

Kramer et al. [38] observed that $96 \%$ of patients referred from primary care to specialised care are diagnosed as suffering from OSA. In a high percentage of these patients, the OSA was severe (mean AHI $44.2 \mathrm{~h}^{-1}$ ) and highly symptomatic (manifest obesity (body mass index (BMI) 37.9 $\left.\mathrm{kg} \cdot \mathrm{m}^{-2}\right)$; chronic snoring $(93 \%)$; excessive hypersomnia (91\%) and witnessed apnoeas (64\%)). The authors concluded that only those patients with a very clear phenotype are referred, and that this does not apply to untypical but very common phenotypes, particularly those related to the cardiovascular system, in women and the elderly [38].

\section{Referrals made mainly by a small number of PCPs}

One of the other conclusions reached by Kramer et al. [38] was that the majority of referrals are made by a limited number of PCPs. Thus, $72 \%$ of the participating PCPs referred only one patient, which means that the remaining $28 \%$ referred $80 \%$ of the patients. The explanation for this discrepancy probably has something to do with heightened awareness of the subject among some professionals, or more comprehensive training/retraining. Similar results were obtained by NAmEN et al. [37] when they put a reminder about collecting sleep data into clinical histories; this was only taken up by $30 \%$ of the participants, who happened to coincide with the ones who made the majority of the referrals to a sleep unit for suspicion of OSA $[37,38]$.

\section{Referral of a very low percentage of patients}

As Netzer and co-workers $[34,39]$ have shown, even though at least $30 \%$ of the population are not sufficient reason to refer a patient in order to rule out OSA.

c) The presence of serious arterial hypertension that does not react to treatment is not sufficient reason to refer a patient for a sleep study if there is no accompanying daytime hypersomnia.

d) The presence of decapacitating hypersomnia of an unknown origin demands an urgent referral for a sleep study, regardless of the other symptoms.

4. The three basic questions that every patient that comes, for whatever reason, to a primary care consulting room must be asked in order to investigate the possibility of obstructive sleep apnoea include: a) Presence of chronic snoring, arterial hypertension and overweight. b) Presence of chronic snoring, witnessed apnoeas and arterial hypertension.

c) Presence of chronic snoring, witnessed apnoeas and daytime hypersomnia. d) Presence of daytime hypersomnia, previous cardiovascular events and obesity.

5. The following are all functions of the primary care physician with respect to the management of patients with OSA, apart from which one?

a) Monitoring of the risk factors for sleep apnoea.

b) Handling of the mild adverse effects of a patient with CPAP.

c) Referral of a patient being treated with CPAP after the reappearance of symptoms.

d) Handling of a patient with CPAP with less than 2 $h$ of compliance every day. 
Table 2 Most important functions of primary care staff with respect to a patient with sleep apnoea or suspected sleep apnoea

$\begin{array}{ll}\text { Detection of patients } & \begin{array}{l}\text { For every patient who sees a PCP } \\ \text { Three key questions: snoring, witnessed apnoeas } \\ \text { and hypersomnia } \\ \text { It is necessary to protocolise referral of patients to } \\ \text { avoid mistakes } \\ \text { Referral protocols }\end{array} \\ \begin{array}{l}\text { Serious hypersomnias, professions entailing risk } \\ \text { and high cardio-respiratory risk }\end{array} \\ \begin{array}{c}\text { Information for the patient } \\ \text { and general population } \\ \text { Control of risk factors } \\ \text { Follow-up of patients with } \\ \text { CPAP }\end{array} & \begin{array}{l}\text { On the risk factors, consequences and treatment of } \\ \text { the dice on diet and general treatment } \\ \text { Common and mild adverse effects } \\ \text { Monitoring of compliance } \\ \text { New referral to a sleep unit }\end{array} \\ & \end{array}$

seeing a PCP present criteria for referral to a sleep unit, the total percentage of referred patients is very small [34, 39]. KRAmer et al. [38] observed that, despite the use of a written reminder from researchers, only $0.13 \%$ of the monitored patients were referred. A similar figure $(0.27 \%)$ was reported by the WallaWalla Project, a training programme undertaken in Washington State, USA [40].

\section{Lack of knowledge of OSA and its consequences}

Although lack of knowledge of this pathology is found in every field, it is not found everywhere in

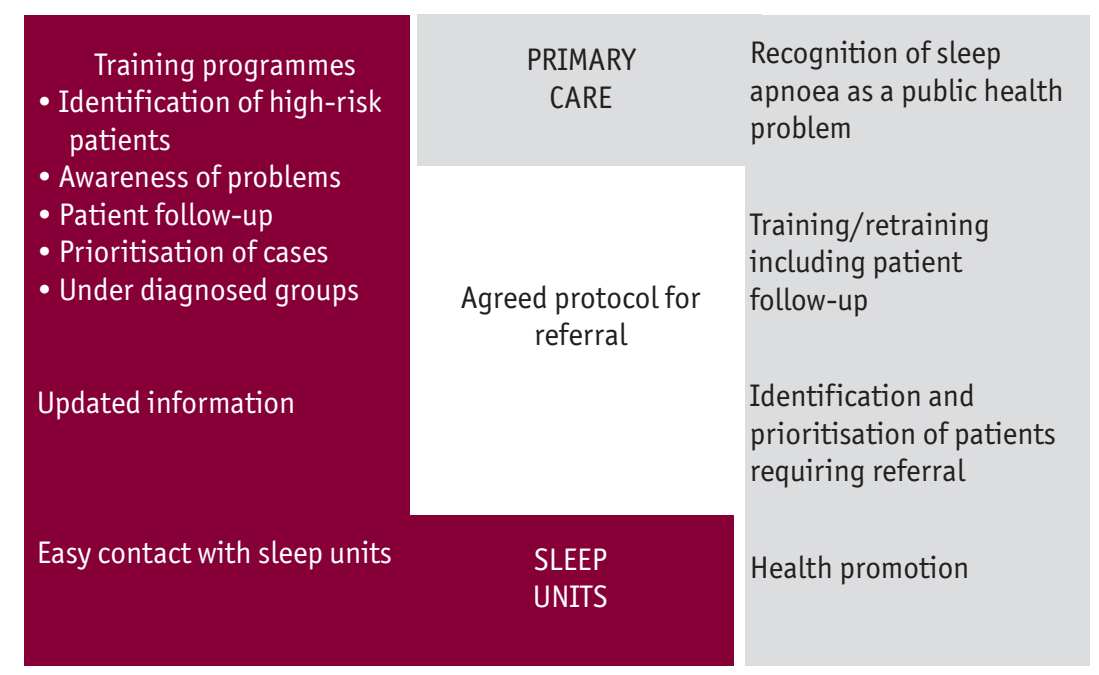

Figure 1

Functions and obligations of primary care and sleep units with respect to the handling of patients with OSA, or suspected of having OSA. equal measure. Most PCPs still consider snoring as a normal situation that coincides with obesity, and daytime hypersomnia as a banal occurrence attributable to age or the medication being taken by the patient. As a study by CHUNG et al. [41] has shown, there is an even greater lack of knowledge of those aspects that have only recently come to light, such as the relationship between OSA and cardiovascular problems and the notion of treatment with CPAP - considered a "treatment for specialists" by PCPs - as a way of solving problems [41].

\section{Relative "youthfulness" of OSA as a disease}

The relationship of smoking and lung cancer, or that of arterial hypertension and cardiovascular events, has been an uncontested given for many decades and so neither the general population nor PCPs could ignore them. OSA is, however, a "young" disease, as the extent of its potential to provoke devastating effects on general health was only discovered a few decades ago. This has meant that the training programmes followed by most PCPs of a certain age did not contemplate this disease, and neither have retraining courses of sufficient quality been set up. Even younger doctors have not spent more than a few hours of training in OSA on their university courses, which translates into the scanty collection of sleeprelated data in the medical histories they compile during their residency.

\section{Lack of knowledge of OSA on the part of the general population}

Finally, there is another vital element that is also missing: knowledge of OSA and its consequences among the general population, as well as awareness of the availability of highly effective treatment. It is difficult to raise awareness in this respect as people traditionally consider the typical symptoms of OSA as normal occurrences that come with age, or with excess weight or the amount of medication they are taking; this means that they do not seek medical advice. BARTLET et al. [42] carried out a study on the general population by sending a letter to 10,000 individuals from the census. These were answered by $35.6 \%$; the prevalence of snoring plus witnessed apnoeas, which would indicate a high clinical probability of OSA, was $6.2 \%$, but only $2.9 \%$ consulted a doctor for this reason (without any significant differences for sex). 


\section{Continuous training about OSA in primary care: the search for solutions}

For all the above reasons, PCPs appear to require retraining, to allow them to handle OSA more effectively in primary care. The conclusions of the Walla-Walla Project -probably the most significant of its kind on training in OSA in primary care - left no room for doubt: training PCPs is an excellent way to increase both the quality and quantity of referrals to a sleep unit for OSA. The Walla-Walla Project established a training programme that included talks and easy contact with sleep units and diagnostic teams. Referrals for suspicion of OSA were evaluated over a 2-yr period and compared with those made in the 2 yrs prior to the training. There was an eightfold increase in the number of referrals of evaluated patients $(0.27 \%$ versus $2.1 \%)$. Among the patients referred for suspicion of OSA, the diagnosis was confirmed in $81 \%$, while $78 \%$ received CPAP and restless leg syndrome was detected in $18 \%$. Furthermore, the quality of the referrals increased considerably, and only a very few were considered beyond the bounds of clinical probability [40].

In this respect, Zozula et al. [43] showed that adherence to an educational programme is sufficient to enable PCPs to quadruple the number of patients they send to a sleep specialist 4 yrs after their involvement in the programme (from $0.06 \%$ to $0.21 \%$ of patients attended in primary care), leading in its turn to almost three times the number of diagnoses of OSA. Similar results were achieved by our work group in a comparable training programme in primary care [44].

Therefore, after taking into account the existing literature, an education programme in OSA in primary care should include, at the very least, training in OSA and its treatment, the provision of the information required in this respect, protocols for referral and prioritisation of patients, flexible contact between PCPs and sleep units and information for the general population. Our view is that such a programme must also be easy for PCPs to understand and complete, avoiding any sophisticated systems they are unlikely to be familiar with, and that it must not take up too much of their time - the heavy workload of the PCP is common knowledge and there is an inverse relationship between the difficulty of a programme and
Table 3 Key questions for every patient who comes to primary care for any reason, in order to identify individuals with a high clinical probability of obstructive sleep apnoea

\section{Do you snore?}

The absence of snoring makes it very difficult to arrive at a diagnosis of OSA. If the answer is yes, or don't know, continue to the following questions

Has anybody remarked to you on any occasion that you stop breathing when you are sleeping?

Isolated apnoeas are very common in snorers, but prolonged or repeated apnoeas witnessed by somebody accompanying the patient are highly indicative of OSA

Do you sleep during the day more easily than you think is normal?

Hypersomnia is also a very common occurrence, but when it is accompanied by snoring or apnoeas, without any apparent cause, or by cardiovascular manifestations it must be investigated, and if it is accompanied by decapacitating forms, association with highrisk professions or a great cardiovascular risk it requires a preferential referral

compliance with it. This type of programme must have the widest possible outreach and, finally, it must give PCPs the sense that it is useful. Crucially, as has already been mentioned, it should be accompanied by an increase in the information on OSA reaching the general population, including the need to consult a PCP so that he or she can instigate a suitable programme.

\section{Actual and future role of primary care in OSA}

It seems obvious, then, that PCPs have to play a more relevant and active role in sleep medicine than they are currently doing, not only by developing a suitable degree of suspicion and actively searching for related clinical signs but also by following up patients who have already been diagnosed and treated. It seems obvious that there is therefore an urgent need to increase

Table 4 Medical history and basic physical examination required for patients in primary care suspected of having OSA

Basic clinical findings: snoring, observed apnoeas and hypersomnia (Epworth Scale) Other symptoms and day- and night-time signs (see table 7)

General history (especially cardiovascular and respiratory findings and medication)

Sleep habits (timetable, siestas, duration, sleep hygiene)

Anthropometric exploration: weight, height, BMI and neck perimeter

Distance from hyoid bone to jaw (short neck)

Facial constitution: maxilla, jawbone, type of bite and evaluation of retro-micrognathia.

Oropharyngoscopy: hypertrophy of soft palate and/or tonsils

Examination using Mallampati Scale

Rhinoscopy: nasal examination (rhinorrhoea, septum, obstruction)

Cardiopulmonary auscultation and measurement of blood pressure 


\section{Protocol for referral of patients}

\begin{tabular}{|c|c|c|c|c|c|c|c|c|}
\hline \multicolumn{9}{|c|}{$\begin{array}{l}\text { Ask every patient (and accompanying person) } \\
\text {-Do you snore? Absence of snoring greatly reduces the probability of sleep apnoea } \\
\text {-Do you get out of breath while you sleep? Repeated, witnessed apnoeas are characteristics } \\
\text { of obstructuve sleep apnoea } \\
\text {-Do you fall asleep in situations where you shouldn't do so? Severe daytime hypersomnia is } \\
\text { "in itself" a sufficient reason for referral }\end{array}$} \\
\hline \multicolumn{5}{|c|}{\begin{tabular}{|l|l|} 
& No
\end{tabular}} & Som & & Often & Don't know \\
\hline \multicolumn{9}{|l|}{ Snoring } \\
\hline \multicolumn{9}{|c|}{ Witnessed apnoeas } \\
\hline \multicolumn{9}{|c|}{ Choking } \\
\hline \multicolumn{9}{|c|}{ Unrefreshing sleep } \\
\hline & & & & Milc & & & derate & Severe \\
\hline \multicolumn{9}{|l|}{ Hypersomnia } \\
\hline \multicolumn{9}{|l|}{ Obesity } \\
\hline \multicolumn{9}{|c|}{$\begin{array}{l}\text { Mild: Usually falls asleep watching TV, reading or as a passenger } \\
\text { Moderate: Usually falls asleep in concerts, church, cinema, theatre, etc... } \\
\text { Severe: Usually falls asleep driving, talking, eating, etc. }\end{array}$} \\
\hline \multicolumn{3}{|c|}{\begin{tabular}{l|l} 
& No \\
\end{tabular}} & \multicolumn{5}{|c|}{ Yes } & Don't know \\
\hline \multicolumn{2}{|c|}{$\begin{array}{l}\text { Arterial } \\
\text { hypertension }\end{array}$} & & \multicolumn{3}{|c|}{$\begin{array}{l}\text { Controlled } \\
\text { Uncontrolled }\end{array}$} & \multicolumn{2}{|c|}{$\begin{array}{l}\text { Resistant or } \\
\text { refractory }\end{array}$} & \\
\hline \multicolumn{2}{|l|}{$\begin{array}{l}\text { Previous CV } \\
\text { events }\end{array}$} & & \multicolumn{3}{|c|}{ Only one } & & Several & \\
\hline \multicolumn{9}{|c|}{$\begin{array}{l}\text { Difficult-to-control hypertension: Blood pressure over } 140 / 90 \mathrm{mmHg} \text { despite use of at least } 3 \\
\text { anti-hypertensive drugs. } \\
\text { Previous CV (cardiovascular) events: Ischemic heart disease, heart failure, cardiac arrhythmias or } \\
\text { stroke. }\end{array}$} \\
\hline \multirow[b]{2}{*}{$\begin{array}{l}\text { Risk } \\
\text { profession }\end{array}$} & & & & \multicolumn{4}{|c|}{ Habitual driver } & Profession \\
\hline & & & & & & & & \\
\hline
\end{tabular}

\begin{tabular}{|ll}
\hline & Type Of Request \\
Preferential & Ordinary \\
Severe hypersomnia & $\begin{array}{l}\text { Habitual snoring with } \\
\text { another finding suggesting sleep apnoea }\end{array}$ \\
$\begin{array}{ll}\text { Justified suspicion of sleep } & \text { Cardiovascular events along with } \\
\text { apnoea in people at risk } & \text { symptoms suggesting sleep apnoea } \\
\text { Resistant or refractory hypertension } & \text { Hypersomnia not explained by other } \\
\text { Snoring, obesity or isolated sytemic hypertension not to be referred }\end{array}$ \\
\hline
\end{tabular}

Figure 2

Protocol for referral of OSA in primary care 
Table 5 Possible adverse effects of CPAP that can be resolved in primary care

\begin{tabular}{|c|c|}
\hline Adverse effects & Treatment \\
\hline $\begin{array}{l}\text { Nasal congestion } \\
\text { or obstruction }\end{array}$ & $\begin{array}{l}\text { Decongestants; nasal steroids; ipratropium bromide, suitable humidification and room } \\
\text { temperature. ENT evaluation if there is no improvement }\end{array}$ \\
\hline $\begin{array}{l}\text { Irritation of the } \\
\text { skin }\end{array}$ & Local protection; change mask. Topical treatment for atopical dermatitis \\
\hline Dry pharynx & Hydration; humidification \\
\hline Noise & Put generator on the floor; avoid leaks \\
\hline Conjunctivitis & Adjust mask \\
\hline Headache & Analgaesics before going to bed \\
\hline Epistaxis & Humidification and adjustment of room temperature. ENT evaluation if persistent \\
\hline Cold & Suitable room temperature; humidifier-heater \\
\hline Insomnia & $\begin{array}{l}\text { Psychological measures for adaptation to the use of CPAP; pressure ramp; mild, non- } \\
\text { benzodiazepine anxiolytic }\end{array}$ \\
\hline Aerophagia & Psychological measures for adaptation to CPAP; mild anxiolytics; postural measures \\
\hline Claustrophobia & Psychological adaptation measures \\
\hline
\end{tabular}

the number of programmes that provide both specific training in sleep disorders in primary care and information to the general population. It is also imperative to find ways to coordinate these programmes with hospital specialists, scientific societies, health authorities and suppliers in order to achieve the necessary training, infrastructure and finance. A PCP should fulfil the several functions with respect to OSA considered as a public health problem (table 2).

\section{Acquisition of knowledge and regular retraining}

Recent studies have shown that, despite the fact that suspicion of OSA is one of the most frequent reasons for referral to a respiratory disease specialist, there is a low degree of clinical suspicion on the part of PCPs $[45,46]$. This is probably due to the relative scientific youthfulness of this disease, limited access to wellgrounded information and the belief that key symptoms of OSA, such as chronic snoring and hypersomnia, can be caused by problems deemed unworthy of referral. So the prototypical referred patient tends to correspond to the most typical forms of OSA [38], which means that the atypical forms - those related to cardiovascular manifestations, and even OSA in women - go more unnoticed. Furthermore, a limited number of PCPs - probably those with the greatest awareness of the subject - are responsible for most of the referrals that are made. This makes it essential for primary care staff to commit themselves to high-quality training/retraining in sleep problems in general, and in OSA in particular (figure 1).

\section{Table 6 Advice related to risk factors for sleep apnoea in primary care}

$\begin{array}{ll}\text { Sleep hygiene } & \text { See Table } 8 \\ \text { Obesity } & \text { Slimming } \\ & \text { Bariatric surgery } \\ \text { Tlcohol } & \text { To be avoided at least } 6 \mathrm{~h} \text { before sleeping } \\ \text { Tobacco } & \text { To be avoided at least } 6 \mathrm{~h} \text { before sleeping } \\ \text { Medication } & \text { Primise the consumption of benzodiazepines } \\ & \text { Hypothyroidism } \\ \text { Associated diseases } & \text { Gastro-oesophageal reflux } \\ \text { Body position } & \text { Avoid supine position: } \\ & \text { Obstacles in the back } \\ & \text { Headrest at } 30^{\circ} \\ \text { Nasal obstruction } & \text { Decongestants } \\ & \text { Nasal corticoids } \\ \text { Ipratropium bromide }\end{array}$


Table 7 Most frequent daytime and night-time symptoms of OSA identifiable in a primary care consulting room

$\begin{array}{ll}\text { Daytime symptoms } & \text { Night-time symptoms } \\ & \\ \text { Excessive daytime sleepiness } & \text { Snoring } \\ \text { Sense of unrefreshing sleep } & \text { Observed apnoeas } \\ \text { Chronic tiredness } & \text { Choking episodes } \\ \text { Morning headaches } & \text { Abnormal movements } \\ \text { Irritability } & \text { Diaphoresis } \\ \text { Apathy } & \text { Frequent arousals } \\ \text { Depression } & \text { Nicturia (adults) and enuresis (children) } \\ \text { Difficulty in concentrating } & \text { Nightmares } \\ \text { Memory loss } & \text { Restless sleep } \\ \text { Reduced sex drive } & \text { Insomnia } \\ \text { Personality changes } & \text { Gastro-oesophageal reflux }\end{array}$

\section{Detection of cases}

It has been estimated that up to $80 \%$ of individuals see their PCP at some point, and most of these do so several times over the course of a year. These occasions, regardless of the reason bringing the individual into the consulting room, are ideal for asking patients a few quick, simple key questions (table 3) that provide clues as to the need to keep on probing (table 4), just as questions are asked for a medical history about whether patients smoke or have smoked in the past, or whether they suffer from hypertension.

\section{Prioritisation and protocolisation of referrals}

One fundamental aspect of a PCP's task is to know how, and with what degree of priority, to refer a patient. This makes it essential to have a simple, informative protocol for referral that does not take up too much time, is based on a mutual agreement between PCPs and a sleep unit and covers the basic characteristics of a patient suspected of having OSA. This protocol must be complete enough to make it possible to classify a patient as having a high, medium or low clinical probability of OSA and, acting on this information, allow a PCP to refer a patient to a sleep unit on an ordinary, preferential or urgent basis (figure 2).

\section{Handling of general treatment and basic problems with CPAP: CPAP is not a specialist treatment}

Despite received opinion to the contrary, CPAP is not a specialist treatment. The large number of patients now equipped with this device, and the ease with which many of their problems can be solved, means that most sleep units do not have sufficient capacity or time to tackle this problem and so a PCP has to be involved. He or she must try to solve some of the most common adverse effects of CPAP, while also being aware of when it is necessary to send a patient to a sleep unit to deal with other more serious adverse effects, or a lack of any effect at all from the usual treatment (table 5).

\section{Table 8 Advice on sleep hygiene that can be given in primary care}

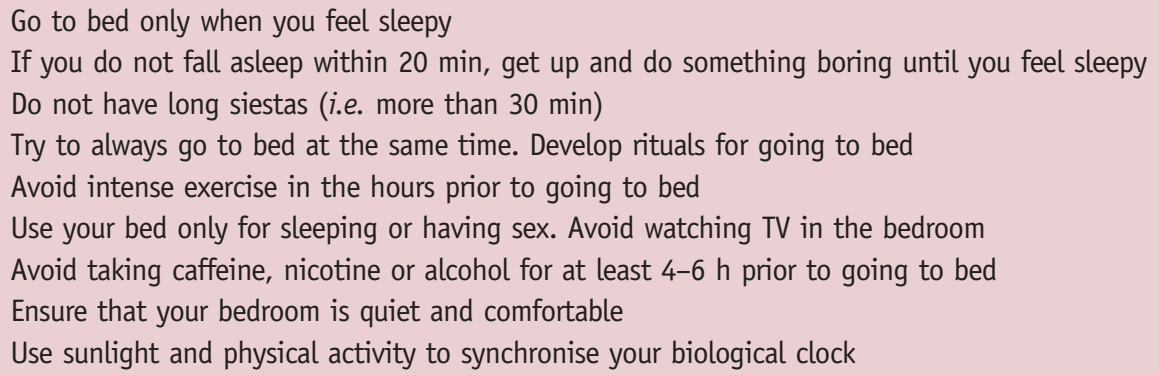




\section{Follow-up of patients with OSA or risk factors for suffering from it}

Once a patient has been diagnosed with OSA, with or without treatment with CPAP, or has been established as having risk factors for OSA without actually having the disease, a PCP must instigate a regular follow-up in order to evaluate certain factors:

- Adverse effects of CPAP. Either for an immediate solution or to refer a patient to the corresponding sleep unit (table 5).

- Non-compliance with the treatment. Search for the cause, referral if necessary.

- Changes in the risk factors for OSA, with special attention to changes in weight or treatment with psychotropics (table 6).

- Reappearance of symptoms of OSA, despite the application of treatment (table 7).
- Medical advice on diet and sleep hygiene (table 8).

\section{Health promotion}

One of the key aspects of handling OSA is information for the general population. A PCP must inform the public about OSA from a general viewpoint, with a special emphasis on the risks associated with it and the alarm mechanisms that should occasion a visit to the doctor. As a result of the high frequency in the general population of the symptoms and signs of OSA - such as chronic snoring, hypersomnia and obesity - these symptoms can pass unnoticed or can be accepted as normal or the result of ageing, and are consequently not reported to a doctor. At the present time this is probably the most significant reason for the lack of diagnosis.

\section{References}

1. Naresh M, Punjabi T. The epidemiology of adult obstructive sleep apnea. Proc Am Thorac Soc 2008; 5: $136-143$.

2. Young T, Palta M, Dempsey J, Skatrud J, Weber S, Badr S. The occurrence of Sleep Disorders Breathing among middle age adults. N Engl J Med 1993; 328: 1230-1236.

3. Young T, Shahar E, Nieto J, Redline S, Newman AB, Gottlieb DJ, et al. Predictors of sleep-disordered breathing in community-Dwelling adults. The Sleep Heart Health Study. Arch Intern Med 2002; 162: 893-900.

4. Martínez-García MA, Soler-Cataluña JJ, Román-Sánchez P, González V, Amorós C, Montserrat JM. Obstructive sleep apnea has little impact on quality of life in the elderly. Sleep Med 2009; 10: 104-111.

5. Terán Santos J, Jimenez Gomez A, Cordero Guevara JA. The association between sleep apnea and the risk of traffic accidents. N Engl J Med 1999; 340: 847-851.

6. Barbe F, Pericas J, Muñoz A, Findley L, et al. Automobile accidents in patients with sleep apnea syndrome. Am J Respir Crit Care Med 1998; 158: 18-22.

7. Masa JF, Rubio M, Findley LJ. Habitually sleepy drivers have a high frequency of automobile crashes associated with respiratory disorders during sleep. Am J Respir Crit Care Med 2000; 162: 1407-1412.

8. Somers VK, White DP, Amin R, et al. Sleep apnea and cardiovascular disease: an American Heart Association/ American College of Cardiology Foundation Scientific Statement from the American Heart Association Council for High Blood Pressure Research Professional Education Committee, Council on Clinical Cardiology, Stroke Council, and Council On Cardiovascular Nursing. Circulation 2008; 118: 1080-1111.

9. Tanikawa T, Horie S, Sakurai S, Iso H. Screening for sleep-disorders breathing at workplaces. Industrial Health 2005; 43: 53-57.

10. Shahar E, Whitney CW, Redline S, et al. Sleep-disordered breathing and cardiovascular disease: cross-sectional results of the Sleep Heart Health Study. Am J Respir Crit Care Med 2001; 163: 19-25.

11. Lavie P, Lavie L, Herer P. All cause mortality in men with sleep apnea syndrome: declining mortality rates with age. Eur Respir $\mathrm{J}$ 2005; 27: 1-7.

12. George CF. Reduction in moor vehicle collisions following treatment of sleep apnoea with nasal CPAP. Thorax 2001; 56: 508-512.

13. Peppard PE, Young T, Palta M, Skatrud J. Prospective study of the association between sleep-disordered breathing and hypertension. N Engl J Med 2000; 342: 1378-1384.

14. Barbé F, Durán-Cantolla J, Capote F, et al. Long-term effect of continuous positive airway pressure in hypertensive patients with sleep apnea. Am J Respir Crit Care Med 2010; 181: 718-726.

15. Haentjens P, Van Meerhaeghe A, Moscariello A, et al. The impact of continuous positive airway pressure on blood pressure in patients with obstructive sleep apnea syndrome: evidence from a meta-analysis of placebo-controlled randomized trials. Arch Intern Med 2007; 167: 757-764.

16. Martínez-García MA, Galiano-Blancart R, Román-Sánchez P, Soler-Cataluña JJ, Cabero-Salt L, Salcedo-Maiques E. Continuous positive airway pressure in sleep apnea prevents new vascular events alter ischemic stroke. Chest 2005; 128: 2123-2129.

17. Good DC, Henkle JQ, Gelber D, Welsh J, Verhulst S. Sleep-disordered breathing and poor functional outcome after stroke. Stroke 1996; 27: 252-259.

18. Parra 0, Arboix A, Montserrat JM, Quinto L, Bechich S, García-Eroles L. Sleep-related breathing disorders: impact on mortality of cerebrovascular disease. Eur Respir J 2004; 24: 267-272.

19. Martínez-García MA, Soler-Cataluña JJ, Ejarque-Martínez L, et al. Continuous positive airway pressure treatment reduces mortality in patients with ischemic stroke and obstructive sleep apnea: a 5-year follow-up study. Am $\mathrm{J}$ Respir Crit Care Med 2009; 180: 36-41. 
20. Lüthje L, Andreas S. Obstructive sleep apnea and coronary artery disease. Sleep Med Rev 2008; 12: 19-31.

21. Shamsuzzaman AS, Gersch BJ, Somers VK. Obstructive sleep apnea: implications for cardiac and vascular disease. JAMA 2003; 290: 1906-1914.

22. Bradley TD, Logan AG, Kimoff RJ. Continuous positive airway pressure for central sleep apnea and heart failure. $N$ Engl J Med 2005; 353: 2025-2033.

23. Young T, Finn L, Peppard P, et al. Sleep-disordered-Breathing and mortality; Eighteen-year follow-up of Wisconsin Sleep Cohort. Sleep 2008; 31: 1071-1078.

24. Marín JM, Carrizo SJ, Vicente E, Agusti AG. Long-term cardiovascular outcomes in men with obstructive sleep apnoea-hypopnoea with or without treatment with continuous positive airway pressure: an observational study. Lancet 2005; 365: 1046-1053.

25. Siccoli MM, Pepperell JC, Kohler M, Craig SE, Davies RJ, Stradling JR. Effects of continuous positive airway pressure on quality of life in patientswith moderate to severe obstructive sleep apnea: data from a randomizedcontrolled trial. Sleep 2008; 31: 1551-1558.

26. Masa JF, Barbé F, Capote F, et al. Recursos y demoras en el diagnóstico del síndrome de apneas-hipopneas durante el sueño. Arch Bronconeumol 2007; 43: 188-198.

27. Punjabi NM. The epidemiology of obstructive sleep apnea. Proc Am Thorac Soc 2008; 5: 136-143.

28. Durán J, Esnaola S, Rubio R, Iztueta A. Obstructive sleep apnea-hypopnea and related clinical features in a population-based sample of subjects aged 30 to 70 years. Am J Respir Crit Care Med 2001; 163: 685-689.

29. Wright J, Sheldon T. Sleep apnoea and its impact on public health. Thorax 1998; 53: 410-413.

30. Phillipson EA. Sleep apnea. A major public health problem. N Engl J Med 1993; 328: 1271-1273.

31. McNicholas WT. Diagnosis of obstructive sleep apnea in adults. Proc Am Thorac Soc 2008; 5: 154-160.

32. Guest JF, helter MT, Morga A, Stradling JR. Cost-effectiveness of using continuous positive airway pressure in the treatment of severe obstructive sleep apnoea/hypopnoea syndrome in the UK. Thorax 2008; 63: 860-865.

33. Ayas NT, Fitzgerald M, Fleetham JA, et al. Cost-effectiveness of continuous positive airway pressure therapy for moderate to severe obstructive sleep apnea/hyopopnea. Arch Intern Med 2006; 166: 977-984.

34. Netzer NC, Hoegel JJ, Loube D, et al. Prevalence of symptoms and risk of sleep apnea in primary care. Chest 2003; 124: 1406-1414.

35. Namen AM, Dunagan DP, Fleischer A, et al. Increased physician-reported sleep apnea: The national ambulatory medical care survey. Chest 2002; 121: 1741-1747.

36. Reuveni H, Tarasiuk A, Wainstock T, Ziv A, Elhayany A, Tal A. Awareness level of obstructive sleep apnea syndrome during routine unstructured interviews of a standardized patient by primary care physicians. Sleep 2004; 27: 15181525.

37. Namen AM, Wymer A, Case D, Haponik EF. Performance of sleep histories in an ambulatory medicine clinic: Impact of simple chart reminders. Chest 1999; 116: 1558-1563.

38. Kramer NR, Cook TE, Carlisle CC, Corwin RW, Millman RP. The role of the primary care physician in recognizing obstructive sleep apnea. Arch Intern Med 1999; 159: 956-968.

39. Netzer NC, Stoohs RA, Metzer CM, et al. Using trhe Berlin Questionnaire to identify patients at risk for the sleep apnoea syndrome. Ann Intern Med 1999; 131: 485-491.

40. Ball EM, Simon RD, Tall AA, Banks MB, Nino-Murcia G, Dement WC. Diagnosis and treatment of sleep apnea within the community. The Walla Walla Proyect. Arch Intern Med 1997; 157: 419-424.

41. Chung SA, Jairam S, Hussain MR, Shapiro CM. Knowledge of sleep apnea in a sample grouping of primary care physicians. Sleep Breath 2001; 5: 115-121.

42. Bartlett DJ, Marshall NS, Williams A, et al. Predictors of primary medical care consultation for sleep disorders. Sleep Med 2008; 9: 857-864.

43. Zozula R, Rosen RC, Jahn EG. Recognition of sleep disorders in a community-based setting following an educational intervention. Sleep Med 2005; 6: 55-61.

44. Martínez-García MA, Soler-Cataluña JJ, Román P, et al. Eficacia de un plan de formación en atención primaria sobre el síndrome de apneas-hipopneas durante el sueño. Arch Bronconeumol 2008; 44: 15-21.

45. Rosen RC, Zozula R, Jahn EG, et al. Low rates of recognition of sleep disorders in primary care: comparison of a community-based versus clinical academic setting. Sleep Med 2001; 2: 47-55.

sıәмsue pə7sә66ns

46. Zozula R, Rosen RC, Jahn EG. Recognition of sleep disorders in a community-based setting folowing an educatinal intervention. Sleep Med 2005; 6: 55-61. 